\title{
DIRICHLET PROBLEMS FOR SINGULAR ELLIPTIC EQUATIONS. II
}

\author{
CHI YEUNG LO
}

ABSTRaCt. Consider an elliptic equation

$$
L[u]=\sum_{i, j=1}^{n} a_{i j} \frac{\partial^{2} u}{\partial x_{i} \partial x_{j}}+\sum_{i=1}^{n} b_{i} \frac{\partial u}{\partial x_{i}}+c u=f
$$

in a bounded domain $G$ in the half space $x_{n}>0$ with boundary $\partial G$ $=S_{1} \cup S_{2}$ of class $C^{2+\alpha}$ where $S_{1}$ is contained in the hyperplane $x_{n}=0$ and $S_{2}$ lies entirely in $x_{n}>0$. The coefficient $b_{n}$ possesses certain type of singularity at $x_{n}=0$. Let $b_{n}=h / k$ where $h \in C^{\alpha}(\bar{G})$ and $k \rightarrow 0$ as $x_{n} \rightarrow 0$. It is found that the solvability of the Dirichlet problem of $L[u]=f$ in $G$ depends on the nature of singularity of $b_{n}$ and also the value of $h$ at $x_{n}=0$.

Consider a class of second order linear partial differential equations

$$
L[u]=\sum_{i, j=1}^{n} a_{i j} \frac{\partial^{2} u}{\partial x_{i} \partial x_{j}}+\sum_{i=1}^{n} b_{i} \frac{\partial u}{\partial x_{i}}+c u=f, \quad b_{n}=\frac{h}{k}
$$

in a bounded domain $G$ in the half space $x_{n}>0$ with boundary $\partial G$ $=S_{1} \cup S_{2}$ of class $C^{2+\alpha}$ where $S_{1}$ is contained in the hyperplane $x_{n}=0$ and $S_{2}$ lies entirely in $x_{n}>0$. We assume (A) the coefficients of (1), with the exception of $b_{n}$, are Holder continuous functions of $\bar{x}=\left(x_{1}, x_{2}, \ldots, x_{n}\right)$ in $\bar{G}$ with exponent $\alpha$, and are bounded by a constant $K$, and $c \leqslant 0$ in $\bar{G}$, and (B) $L[u]$ is uniformly elliptic in $\bar{G}$, i.e. there exists a positive constant $m$ such that

$$
\sum_{i, j=1}^{n} a_{i j}(\bar{x}) \xi_{i} \xi_{j} \geqslant m \sum_{i=1}^{n} \xi_{i}^{2}
$$

for all $n$-tuples of real numbers $\left(\xi_{1}, \ldots, \xi_{n}\right)$ and all $\bar{x} \in \bar{G}$. By normalization, $a_{n n}(\bar{x})=1$.

We would like to investigate whether the Dirichlet problem of (1) is solvable in $G$, subject to the condition that the coefficient $b_{n}$ possesses certain type of singularity at $x_{n}=0$. The case $b_{n}(\bar{x})=h(\bar{x}) / x_{n}$, where $h(\bar{x})$ is Holder continuous in $\bar{G}$, has been treated in [2]. It turns out that an existence and unique theorem for the Dirichlet problem of

Received by the editors October 14, 1974.

AMS (MOS) subject classifications (1970). Primary 35J70, 35J25.

Key words and phrases. Singular elliptic equations, boundary value problem, barrier function.

- American Mathematical Society 1976 


$$
\sum_{i, j=1}^{n} a_{i j} \frac{\partial^{2} u}{\partial x_{i} \partial x_{j}}+\sum_{i=1}^{n-1} b_{i} \frac{\partial u}{\partial x_{i}}+\frac{h}{x_{n}} \frac{\partial u}{\partial x_{n}}+c u=f
$$

in $G$ is proved for $h\left(x_{1}, x_{2}, \ldots, x_{n-1}, 0\right)<1$ whereas uniqueness theorem is established for (3) when the boundary data is only given in $S_{2}$ for $h\left(x_{1}, x_{2}, \ldots, x_{n-1}, 0\right) \geqslant 1$. These theorems indicate that the behavior of $b_{n}(\bar{x})$ at $x_{n}=0$ plays an important part in the formulation of boundary value problems of (3) in $G$. In the general case, we may expect that the solvability of the Dirichlet problem depends not only on the value of $h(\bar{x})$ at $x_{n}=0$, but also the nature of singularity of $b_{n}(\bar{x})$ when $x_{n} \rightarrow 0$.

THEOREM 1. Suppose the coefficients of (1) satisfy hypotheses (A) and (B), and $\phi(\bar{x})$ is any given continuous function on $\partial G$. Then there exists a unique solution $u \in C^{2+\alpha}(G) \cap C^{0}(\bar{G})$ of $(1)$ with $u=\phi$ on $\partial G$ if $h(\bar{x})$ also satisfies hypothesis (A) and any one of the following conditions is fulfilled.

(i) $b_{n}(\bar{x})=h(\bar{x}) \log x_{n}$,

(ii) $b_{n}(\bar{x})=h(\bar{x}) x_{n}^{-m}, m \geqslant 2$, a positive integer and $h\left(x_{1}, \ldots, x_{n-1}, 0\right)<0$,

(iii) $b_{n}(\bar{x})=h(\bar{x}) \exp \left(1 / x_{n}\right)$ and $h\left(x_{1}, \ldots, x_{n-1}, 0\right)<0$.

Remarks. The function $b_{n}(\bar{x})$ in each case represents different type of singularity when $x_{n} \rightarrow 0$. This indicates a close relationship between the growth of $b_{n}(\bar{x})$ and the values of $h(\bar{x})$ on $x_{n}=0$.

The approach and the arguments for the proof of this theorem in each case is the same as those used in the proof of Theorem 2 in [2], which represents the special case $k(\bar{x})=x_{n}$ and $h\left(x_{1}, \ldots, x_{n-1}, 0\right)<1$. The key point in the proof is to construct a barrier function $v(\bar{x})$ for any point $Q=\bar{x}^{0}=\left(x_{1}^{0}\right.$, $\left.x_{2}^{0}, \ldots, x_{n-1}^{0}, 0\right) \in S_{1}$ with the following properties: (a) $v(\bar{x})$ is continuous in a sufficiently small neighborhood $W_{Q}=\left\{\bar{x}|| \bar{x}-\bar{x}_{0} \mid<\rho, x_{n} \geqslant 0\right\}$; (b) it vanishes at $Q ;$ (c) it is positive in $W_{Q} \backslash Q$; and (d) $L[v]<-1$. If the construction of the barrier function is done, then, by using Schauder's Lemma [3], [4] and Hopf's maximum principle [1] for regular elliptic equations, we can arrive at the conclusion. Hence, we shall confine ourselves to the explicit construction of the barrier function in each case, and omit all the details. Readers are requested to refer to [2] for a complete proof.

Cases (i) and (iii). Let

$$
v(\bar{x})=x_{n}^{\beta}+\sum_{i=1}^{n-1}\left(x_{i}-x_{i}^{0}\right)^{2}, \quad 0<\beta<1 .
$$

Then

$$
\begin{aligned}
L[v]= & 2\left(a_{11}(\bar{x})+\cdots+a_{n-1, n-1}(\bar{x})\right)+\beta(\beta-1) x_{n}^{\beta-2} \\
& +2\left(b_{1}(\bar{x})\left(x_{1}-x_{1}^{0}\right)+\cdots+b_{n-1}(\bar{x})\left(x_{n-1}-x_{n-1}^{0}\right)\right) \\
& +\beta b_{n}(\bar{x}) x_{n}^{\beta-1}+c(\bar{x}) v(\bar{x}) \\
< & 2(n-1) K+2 \rho(n-1) K+\beta\left[\beta-1+b_{n}(\bar{x}) x_{n}\right] x_{n}^{\beta-2} .
\end{aligned}
$$


For $b_{n}(\bar{x})=h(\bar{x}) \log x_{n}$, we have, by the continuity of $h(\bar{x}), h(\bar{x}) x_{n} \log x_{n}$ goes to zero uniformly as $x_{n} \rightarrow 0$. The term $\beta(\beta-1) x_{n}^{\beta-2}$ dominates the right side of (4). Hence we have $L[v]<-1$ for sufficiently small values of $\rho$.

For $b_{n}(\bar{x})=h(\bar{x}) \exp \left(1 / x_{n}\right)$ and $h\left(x_{1}, \ldots, x_{n-1}, 0\right)<0, x_{n}^{\beta-1} \exp \left(1 / x_{n}\right)$ goes to infinity uniformly as $x_{n} \rightarrow 0$. Hence, in a sufficiently small neighborhood of $Q$, the term $\beta \exp \left(1 / x_{n}\right) h(\bar{x}) x_{n}^{\beta-1}$ dominates the right side of (4), and its sign depends on the sign of $h\left(x_{1}, \ldots, x_{n-1}, 0\right)$. Hence, by continuity, we have $L[v]<-1$ for sufficiently small values of $\rho$. $<0$.

Case (ii). $b_{n}(\bar{x})=h(\bar{x}) x_{n}^{-m}, m \geqslant 2$, a positive integer and $h\left(x_{1}, \ldots, x_{n-1}, 0\right)$

Let

$$
v(x)=x_{n}^{\lambda}+\sum_{i=0}^{n-1}\left(x_{i}-x_{i}^{0}\right)^{2}
$$

where $\lambda$ is chosen such that $2 \leqslant m \leqslant \lambda<m+1$. Then

$$
L[v]<2(n-1) K+2 \rho(n-1) K+\lambda(\lambda-1) x_{n}^{\lambda-2}+\lambda h(\bar{x}) x_{n}^{\lambda-m-1} .
$$

Since $h\left(x_{1}, x_{2}, \ldots, x_{n-1}, 0\right)<0$, we have, by continuity, $L[v]<-1$ for sufficiently small values of $\rho$.

Hence the barrier function in each case has properties (a)-(d).

For $b_{n}(\bar{x})=h(\bar{x}) x_{n}^{-m}, m \geqslant 2$, and $h\left(x_{1}, \ldots, x_{n-1}, 0\right)>0$ we have the following uniqueness theorem.

THeOREM 2. Let $b_{n}(\bar{x})=h(\bar{x}) x_{n}^{-m}, m \geqslant 2$ and $h\left(x_{1}, \ldots, x_{n-1}, 0\right)>0$. Then there is at most one solution $u(\bar{x})$ of (1) which is regular in $G$, remains bounded when $x_{n} \rightarrow 0$, and assumes given continuous values on the boundary $S_{2}$.

Again the proof of this theorem is exactly the same as shown in Theorem 1 of [2] as long as we can demonstrate the existence of a barrier function $w(\bar{x})$ with the following properties: (a) $w(\bar{x})$ is positive in $\bar{G}$; (b) $w(\bar{x})$ converges uniformly to infinity when $x_{n} \rightarrow 0$ as (c) $L[w]<0$. To this end, we set $w(\bar{x})=x_{n}^{-\beta}-\left(x_{1}-a\right)^{\gamma}+A$ where $2 \leqslant m \leqslant \beta<m+1$ and $a$ is chosen such that $x_{1}-a>1$ for all $\bar{x}=\left(x_{1}, x_{2}, \ldots, x_{n}\right) \in \bar{G}$ whereas $\gamma$ and $A$ are positive constants to be determined later. Then

$$
\begin{aligned}
L[w]= & -a_{11}(\bar{x}) \gamma(\gamma-1)\left(x_{1}-a\right)^{\gamma-2}-b_{1}(\bar{x}) \gamma\left(x_{1}-a\right)^{\gamma-1} \\
& +(-\beta)\left[-\beta-1+h(\bar{x}) x_{n}^{-m+1}\right] x_{n}^{-\beta-2}+c(\bar{x}) w(\bar{x}) \\
< & -m \gamma(\gamma-1)\left(x_{1}-a\right)^{\gamma-2}-\gamma b_{1}(\bar{x})\left(x_{1}-a\right)^{\gamma-1} \\
& +(-\beta)\left(-\beta-1+h(\bar{x}) x_{n}^{-m+1}\right) x_{n}^{-\beta-2}+c(\bar{x}) w(\bar{x}) \\
< & -m \gamma(\gamma-1)-\gamma B+(-\beta)\left[-\beta-1+h(\bar{x}) x_{n}^{-m+1}\right] x_{n}^{-\beta-2} \\
& +c(x) w(x) \quad \text { where } B=\max _{\bar{x} \in \bar{G}}\left|b_{1}(\bar{x})\left(x_{1}-a\right)\right| .
\end{aligned}
$$

Since $h\left(x_{1}, \ldots, x_{n-1}, 0\right)<0$, we have by continuity, choose a $\rho>0$ such 
that $0 \leqslant x_{n} \leqslant \rho,-\beta-1+h(\bar{x}) x_{n}^{-m+1}>0$ uniformly. Then for $\bar{x} \in \bar{G}$ $\cap\left\{\bar{x} \mid x_{n}>\rho\right\}$, choose $\gamma>1$ large enough that

$$
-m \gamma(\gamma-1)-\gamma B+(-\beta)\left[-\beta-1+h(\bar{x}) x_{n}^{-m+1}\right] x_{n}^{-\beta-2}>0 .
$$

For this $\gamma$, we can choose $A$ large enough such that $w(\bar{x})>0$ for all $\bar{x} \in \bar{G}$. With these choices of $\rho, \gamma$ and $A$, we see that $w(\bar{x})$ have all the required properties.

REMARK. The approach in this paper can also be used to study boundary value problems of (1) in a bounded set in the hyperoctant $D=\left\{x_{i}>0, i\right.$ $=1, \ldots, n\}$ with part of its boundary on the hyperplanes $x_{i}=0, i=1, \ldots$, $n$, where the functions $b_{i}(x), i=1, \ldots, n$, become singular. A special case of (1) has been proved by Hall, Quinn, and Weinacht [5] for the equation

$$
L_{\alpha, \beta}[u]=\sum_{i=1}^{n} \frac{\partial^{2} u}{\partial x_{i}^{2}}+\frac{\alpha}{x_{n-1}} \frac{\partial u}{\partial x_{n-1}}+\frac{\beta}{x_{n}} \frac{\partial u}{\partial x_{n}}=0
$$

in the quarter ball $Q_{R}=\left\{|x| \leqslant R, x_{n-1}>0, x_{n}>0\right\}$ for different real values of $\alpha$ and $\beta$ by establishing Poisson integral formulas.

\section{REFERENCES}

1. E. Hopf, Elementare Bemerkungen über die Lösungen partieller Differentialgleichungen vom elliptischen Typus, S.-B. Preuss. Akad. Wiss. 19 (1927), 147-152.

2. C. Y. Lo, Dirichlet problems for singular elliptic equations, Proc. Amer. Math. Soc. 39 (1973), 337-342. MR 47 \# 5443.

3. J. Schauder, Uber lineare elliptische Differentialgleichungen zweiter Ordnung, Math. Z. 38 (1934), 257-282.

4. - Numerische Abschätzungen in elliptischen linearen Differentialgleichungen, Studia Math. 5 (1934), 34-42.

5. N. S. Hall, D. W. Quinn and R. J. Weinacht, Poisson integral formulas in generalized biaxially symmetric potential theory, SIAM J. Math. Anal. 5 (1974), 111-118. MR 49 \#173.

Department of Mathematics, Michigan State University, East Lansing, Michigan 48824 (Current address)

Department of Mathematics, Hong Kong University, Hong Kong 